\title{
User-friendly, magnetically sealed plug-and-play sensor module for online electrochemical sensing for fluidic devices
}

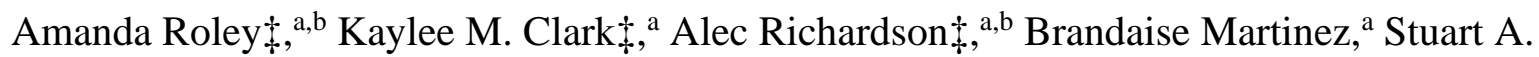 \\ Tobet, ${ }^{\mathrm{b}}$ Charles S. Henry*a,b \\ a Department of Chemistry, Colorado State University, 1872 Campus Delivery, Fort Collins, \\ Colorado, USA \\ ${ }^{\mathrm{b}}$ School of Biomedical Engineering, Colorado State University, Fort Collins, Colorado, USA \\ †. Roley, K. Clark, and A. Richardson contributed equally to this work. \\ *Corresponding author: Charles S. Henry \\ Email: Chuck.Henry@ colostate.edu \\ Phone: (970) 491-2852
}

\begin{abstract}
The growth in fluidic devices, such as organ-on-chip (OOC) technology, comes with a need for growth in sensing capabilities of key biomolecules to help elucidate changes during the time course of experiments. We developed an on-line, easy-to-assemble, 3D-printed electrochemical sensor module that is magnetically sealed for ease of assembly. The sensor module includes a plugand-play format for electrochemical sensors made in finger-tight fittings to allow for a wide selection of experimental set-ups and target molecules. Here, we report the feasibility of the sensor module as well as demonstrate its use for electrochemical sensing with integrated thermoplastic electrodes (TPEs). The sensor module withstood over $300 \mathrm{kPa}$ of backpressure and demonstrated reliable performance with TPEs when using cyclic voltammetry $(\mathrm{CV})$ and amperometry under flow conditions. CVs using the ferri/ferrocyanide $\left(\mathrm{K}_{3 / 4}\left[\mathrm{Fe}(\mathrm{CN})_{6}\right]\right)$ redox system demonstrate that the sensor module does not hinder the expected linear response with respect to analyte concentration. Further CVs and amperometry demonstrated the use of the sensor module under flow conditions. Such success in device design and usability is promising for future work using the on-line sensor module with a variety of applications.
\end{abstract}

\section{Introduction}

Fluidic and microfluidic devices have greatly expanded the toolbox for analytical measurements in countless fields. These devices have been used broadly for applications including single molecule detection, ${ }^{1}$ immunoassays, ${ }^{2}$ environmental analysis,${ }^{3}$ and organ-on-chip (OOC) devices. ${ }^{4}$ In many devices, fractions collectors or off-chip assays are used, which lack the capability to measure changes in analytes with high time resolution and can require large working volumes. For devices that have integrated sensors, in many previously reported devices, if the sensor fails, both the device and the sensor require refabrication. ${ }^{5}$ This process can be expensive and time-consuming, particularly for OOC devices, in which integrated sensors are desirable for monitoring of tissue-specific biochemical processes. ${ }^{6}$

Electrochemical sensors are well suited for integration into fluidic devices because they are highly sensitive and selective, cost-effective, can be readily fabricated at micro scales, and exhibit fast response times. ${ }^{7}$ While some OOC devices feature on-chip electrochemical or optical 
biosensors to monitor changes in environmental parameters (e.g. $\left.\mathrm{O}_{2}, \mathrm{pH}, \mathrm{CO}_{2}\right)^{8-11}$ and metabolic products (e.g. glucose, lactate $)^{12,13}$ in real-time, they often lack reusability and simple integration. Sensor module designs featuring plug-and-play architectures are especially advantageous because individual sensors can be swapped when failures in occur, and microfluidic platforms can be reused in separate experiments requiring different sensors. ${ }^{5}$ Examples of plug-and-play sensor modules include 3D-printed microfluidic devices that accommodate electrochemical sensors housed in finger-tight fittings ${ }^{14}$ or custom holders, ${ }^{15,16}$ as well as a 3D-printed flow cells that accommodate off-the-shelf optical components. ${ }^{6}$

An attractive method for microfluidic device fabrication is $3 \mathrm{D}$ printing because it enables rapid prototyping of different designs in one step, and in some cases, multi-material parts with varying mechanical properties. ${ }^{16,17}$ Channel clogging, a common issue when printing internal microchannels, can be circumvented by 3D printing open-faced microfluidic devices and reversibly or irreversibly sealing the printed parts to various substrates. ${ }^{18-21}$ Reversibly sealed microfluidic devices are especially desirable for OOC applications because they can be disassembled, sterilized, and reused for subsequent experiments. ${ }^{22}$ Different methods have been used to reversibly seal microfluidic devices, such as adhesive bonds, ${ }^{23,}{ }^{24}$ vacuum suction, ${ }^{25,}{ }^{26}$ and mechanical clamps fastened with screws ${ }^{27,} 28$ or magnets. ${ }^{29-31}$ Previous reports of magnetically sealed devices require careful assembly, have comparatively low burst pressures, or have open channels which can easily disturb the chemical conditions of the fluid while measuring. ${ }^{29-31}$ These devices also often rely on PDMS for the channel which can be easily deformed, messy to assemble, and difficult to clean for reuse. ${ }^{29-31}$

Here, we report a plug-and-play sensor module for use in fluidic systems that makes use of magnetic closures to create a sealed device that can operate at relatively high pressures. The microfluidic platform consists of two 3D-printed layers separated by a silicone gasket with defined microfluidic channels. Magnets installed in each 3D printed layer provide high-pressure reversible seals and allow for rapid, self-aligning assembly and disassembly which is highly user friendly The top 3D-printed layer contains threaded ports that accommodate both electrochemical and optical sensors housed in commercial PEEK fingertight fittings. ${ }^{32}$ Importantly, this design reduces fabrication costs since both the microfluidic platform and sensors can be cleaned and reused between experiments. The sensor module's plug-and-play architecture, easy and reversible assembly, and ability to withstand high fluid pressures enables it to be used for a wide variety of applications.

\section{Experimental}

\section{Reagents}

Solutions were prepared using $18.2 \mathrm{M} \Omega \cdot \mathrm{cm}$ water purified using a Milli-Q system (Millipore Sigma, USA). Graphite (Grade 3569) was purchased from Asbury Graphite Mills, INC. (Asbury, NJ). Polycaprolactone (PCL) was purchased from ThermoMorph® (Toledo, OH). Dichloromethane and potassium nitrate (cryst./certified ACS) were purchased from Fisher Chemical (Waltham, MA). Potassium ferricyanide(III) was purchased from Sigma-Aldrich (Saint Louis, MO). Ferrocenylmethyl trimethylammonium $\left(\mathrm{FcTMA}^{+}\right)$was synthesized in house using a previously reported method. ${ }^{33}$ 


\section{Device Design and Fabrication}

Devices (Figure 1) were designed in SolidWorks CAD software (Dassault Systems, Waltham, MA) and the top and bottom layers were 3D-printed with a Form 3 SLA printer (Formlabs, Somerville, MA) using Clear V4 resin. After printing, the devices were soaked in isopropyl alcohol for 20 minutes to remove uncured resin and subsequently post-cured according to Formlabs recommendations. All 3D-printed devices were coated with a silicone-modified conformal coating (MG Chemicals, Surrey, BC) to provide a smooth surface finish and improve optical transparency. N42 Neodymium bar magnets (K\&J Magnets Inc., Pipersville, PA) were installed in slots on the inner surface of each 3D-printed layer using cyanoacrylate glue (Krazy Glue, Elmers Products, High Point, NC), providing $11.5 \mathrm{lbf}$ of total clamping force. The device was intentionally designed such that opposing magnets are nearly in contact after device assembly to maximize the attractive force between magnets. Two distinct microfluidic platforms with different middle layers were designed to demonstrate the versatility of magnetic connections (Figure 1).

In the first design (Figure 1A), the middle layer consists of a 20A durometer silicone gasket (McMaster-Carr, Elmhurst, IL) cut with an Epilog Zing laser cutter to define the microfluidic channel ( $1 \mathrm{~mm}$ width, $200 \mu \mathrm{m}$ height, $33 \mathrm{~mm}$ length). To ensure a consistent channel height, the gasket was compressed by a set percent $(25 \%)$ of its original thickness. The allowable percent compression was estimated by first converting the maximum clamping force provided by the magnets to a clamping pressure using the following equation:

$$
P_{\text {clamp }}=\frac{F_{\text {clamp }}}{S A_{\text {gasket }}}
$$

$\mathrm{P}_{\text {cham }}$ Was subsequently compared to a compression-deflection curve for $20 \mathrm{~A}$ durometer silicone gaskets, which defines the pressure needed to compress a material by a given percent. The top 3Dprinted layer contains threaded inlet/outlet ports and two sensor ports that accommodate 10-32 and 1/4-28 fingertight fittings (IDEX Health and Science, LLC, Oak Harbor, WA), respectively. The bottom 3D printed layer features notches aligned with the sensor ports to prevent installed sensors from occluding the microfluidic channel. Rubber O-rings (McMaster-Carr, Los Angeles, CA) were installed at the base of the inlet/outlet ports to ensure leak-free connections.

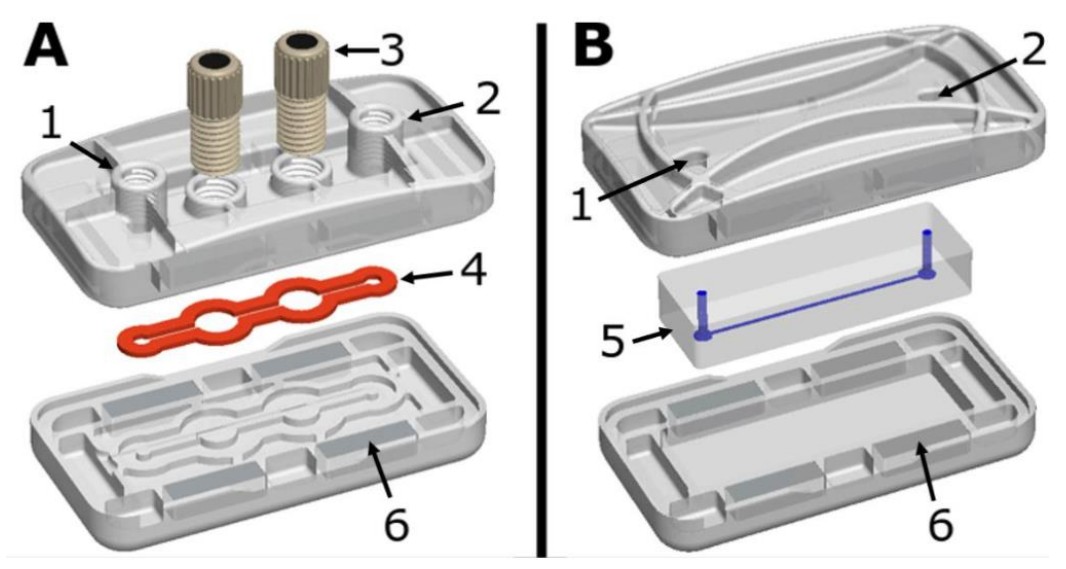

Figure 1. (A) CAD rendering of the magnetically assembled sensor module with the microfluidic channel defined by a laser cut gasket. (B) CAD rendering of alternative device design with microfluidic channel defined by PDMS. 16 indicate the fluid inlet, fluid outlet, electrochemical sensors, gasket, PDMS chip, and magnets, respectively. 
The second design (Figure 1B) uses a middle layer comprised of a PDMS chip with an open- faced microfluidic channel (500 $\mu \mathrm{m}$ width, $200 \mu \mathrm{m}$ height, $33 \mathrm{~mm}$ length). The PDMS chip was fabricated using a 3D-printed mold combined with conventional soft lithographic techniques. ${ }^{34}$ Briefly, Sylgard 184 silicone elastomer base and Sylgard 184 elastomer curing agent were thoroughly mixed at a 10:1 ratio and degassed in a vacuum desiccator. The mixture was then cast over the 3D-printed mold and cured for $24 \mathrm{~h}$ in an $80^{\circ} \mathrm{C}$ oven. A 10:1 elastomer base to curing agent ratio was chosen because higher ratios (e.g., 15:1) resulted in significant channel deformation under compression. Steel connectors (18G) were inserted into biopsy-punched holes at the inlet and outlet to establish fluidic connections.

\section{Leakage Tests}

The sealing performance of each device design was evaluated with two magnet arrangements. Magnets were either installed along the devices' sides parallel to the channel axis (Figure 2, arrangement 1) or around the devices' periphery (Figure 2, arrangement 2) providing more equal spacing. A $60 \mathrm{~mL}$ syringe filled with DI water and food dye was loaded on an NE-300 syringe pump (New Era Pump Systems Inc., Farmingdale, NY) and connected to an assembled device using commercial fittings and tubing. Next, an F-238 NanoTight Capillary sleeve (IDEX Health and Science, LLC, Oak Harbor, WA) was connected to the device's outlet to provide additional fluidic resistance. The fluid was pumped through the device for 30 seconds in 0.5 $\mathrm{mL} / \mathrm{min}$ increments until leakage was observed. This process was repeated for all devices and magnet arrangements.

Volumetric flow rates $(\mathrm{Q})$ were converted to pressure values $(\Delta \mathrm{P})$ using the following equation:

$$
\Delta P=Q R_{\text {total }}
$$

$\mathrm{R}_{\text {toul }}$ is the combined fluidic resistance provided by the microfluidic channel and capillary sleeve. The individual resistance contributions from the microfluidic channel and capillary sleeve were calculated from equations 3 and 4, respectively:

$$
\begin{gathered}
R_{\text {channel }}=\frac{12 \mu L}{w h^{3}\left(1-0.63 \frac{h}{w}\right)} \\
R_{\text {capillary }}=\frac{8 \mu L}{\pi d^{4}}
\end{gathered}
$$

Here, $\mu$ is the dynamic viscosity, $L$ is the channel length, $w$ is the channel width, $h$ is the channel height, and $d$ is the capillary inner diameter.

\section{Electrode Fabrication}

Electrochemical sensors were made by heat-pressing a previously reported thermoplastic electrode (TPE) material ${ }^{35-37}$ into 1/4-28 PEEK fingertight fittings. To fabricate the electrode material, polycaprolactone (PCL) pellets were first dissolved in dichloromethane followed by the addition of graphite at a 3:1 (w/w) graphite to PCL ratio. Once homogenized, the mixture was poured onto a silicon wafer to allow the solvent to evaporate. The dried material was then molded into fingertight fittings using a hydraulic heat press set to $75^{\circ} \mathrm{C}$. Next, the ends of the fingertight fittings were sanded to remove excess material and exfoliate the carbon. Each electrode's resistance was measured with a two-point probe (Fluke 187 multimeter, accuracy of $0.01 \Omega$ ) for 
quality control purposes. Electrical connections were established using electrical wire and silver paint, and epoxy was applied over the connections for mechanical reinforcement. Prior to electrochemical experiments, fingertight fittings were wrapped with Teflon tape to prevent leakage.

\section{Electrochemical Experiments}

The electrodes' electrochemical performance was characterized via flow injection analysis coupled with cyclic voltammetry (CV). A NE-300 syringe pump (New Era Pump Systems Inc., Farmingdale, NY) was used for sample injection while a CHI660b potentiostat (CH Instruments, Inc., Austin, TX) and a CHI832 bipotentiostat (CH Instruments, Inc., Austin, TX) were used for electrochemical measurements. For CV experiments, two fingertight fitting TPEs were screwed into the sensor module's ports as described above to be used as working and counter electrodes. A saturated calomel reference electrode was placed in the downstream waste beaker filled with 1.0 $\mathrm{M}$ potassium nitrate $\left(\mathrm{KNO}_{3}\right)$. For all $\mathrm{CVs}$, the potential was scanned from $0 \mathrm{mV}$ to $600 \mathrm{mV}$ versus an SCE reference with a step of $10 \mathrm{mV}$ and a scan rate of $100 \mathrm{mV} / \mathrm{s}$.

For flow experiments, a solution containing $5 \mathrm{mM}$ potassium ferricyanide(III) $\left(\mathrm{K}_{3}\left[\mathrm{Fe}(\mathrm{CN})_{6}\right]\right)$ in $1 \mathrm{M} \mathrm{KNO}_{3}$ was used for all flow rates. The syringe pump was started, and measurements were taken after approximately 5-10 seconds to ensure stable flow without bubbles. Four flow rates were tested in triplicate, from $0.1 \mathrm{~mL} / \mathrm{min}$ to $0.5 \mathrm{~mL} / \mathrm{min}$ in random order. For the static experiments, 12 concentrations of ferri/ferrocyanide $\left(\mathrm{K}_{3 / 4}\left[\mathrm{Fe}(\mathrm{CN})_{6}\right]\right)$ in $1 \mathrm{M} \mathrm{KNO} \mathrm{KN}_{3}$ were tested in triplicate, from $0 \mathrm{mM}$ to $20 \mathrm{mM}$ in random order using the same fingertight fitting TPE as the working electrode each time. For each measurement, the syringe pump was run for approximately 5-10 seconds to fill and purge the sensor module. CVs were run for each concentration under static conditions.

For flow amperometry experiments, two fingertight fitting TPEs working electrodes were screwed into the sensor module's ports to be used simultaneously with reference and counter electrodes downstream in a waste beaker filled with $1.0 \mathrm{M}$ potassium nitrate $\left(\mathrm{KNO}_{3}\right)$. Potential was held at $+200 \mathrm{mV}$ for the first working electrode and $+400 \mathrm{mV}$ for the second and current of each was measured in real time. Flow was set to $100 \mu \mathrm{L} / \mathrm{min}$ injections of $1 \mathrm{mM} \mathrm{FcTMA}^{+}$in 0.1 $\mathrm{M} \mathrm{KCl}$ were added sequentially.

The sensor module was simply cleaned between experiments by disassembling and rinsing with DI water.

\section{Results and Discussion}

\section{Device Design and Seal Integrity}

The placement of the magnets in the sensor module are arranged in a manner in such a way that once the gasket is placed, the module simply self-aligns. The result is that the sensor module can be assembled correctly in under 5 seconds while still maintaining high quality seals. The ability of the sensor module to withstand backpressures was tested. The sensor module exhibited excellent sealing capabilities across all device designs/magnet arrangements and withstood backpressures over $300 \mathrm{kPa}$ from flow rates over $10 \mathrm{~mL} / \mathrm{min}$ (Figure 2). The burst pressure was significantly higher for devices designed with a gasket material compared to devices designed with a PDMS chip (Figure 2). Various magnet arrangements were tested to determine if they provided more reliable seals. Higher burst pressures were observed in devices with magnets distributed equally around the perimeter (Figure 2, magnet arrangement 2), although the difference was not 
significant for gasketed devices. Magnet arrangement 2 provided more clamping force near the devices' inlets and outlets where leakages typically occurred.

Several groups have reported magnetic clamping systems for cell culture applications with resulting burst pressures as high as $58 \mathrm{kPa} .{ }^{29-31,38}$ Rafat et al. and Occhetta et al. reported devices with even higher burst pressures $(145 \mathrm{kPa}, 150 \mathrm{kPa}) .{ }^{31,39}$ However, all these devices were designed to clamp PDMS chips alone, whereas the reported sensor module can clamp multiple materials. All of the designs/magnet arrangements tested here are suitable for OOC applications because the flow rates in OOC devices are typically an order of magnitude lower than the flow rates used for the leakage tests. Further, regardless of the magnet placement, the sensor module withstood higher backpressures compared to most previously reported microfluidic devices reversibly sealed with magnetic connections. ${ }^{30,31,38}$

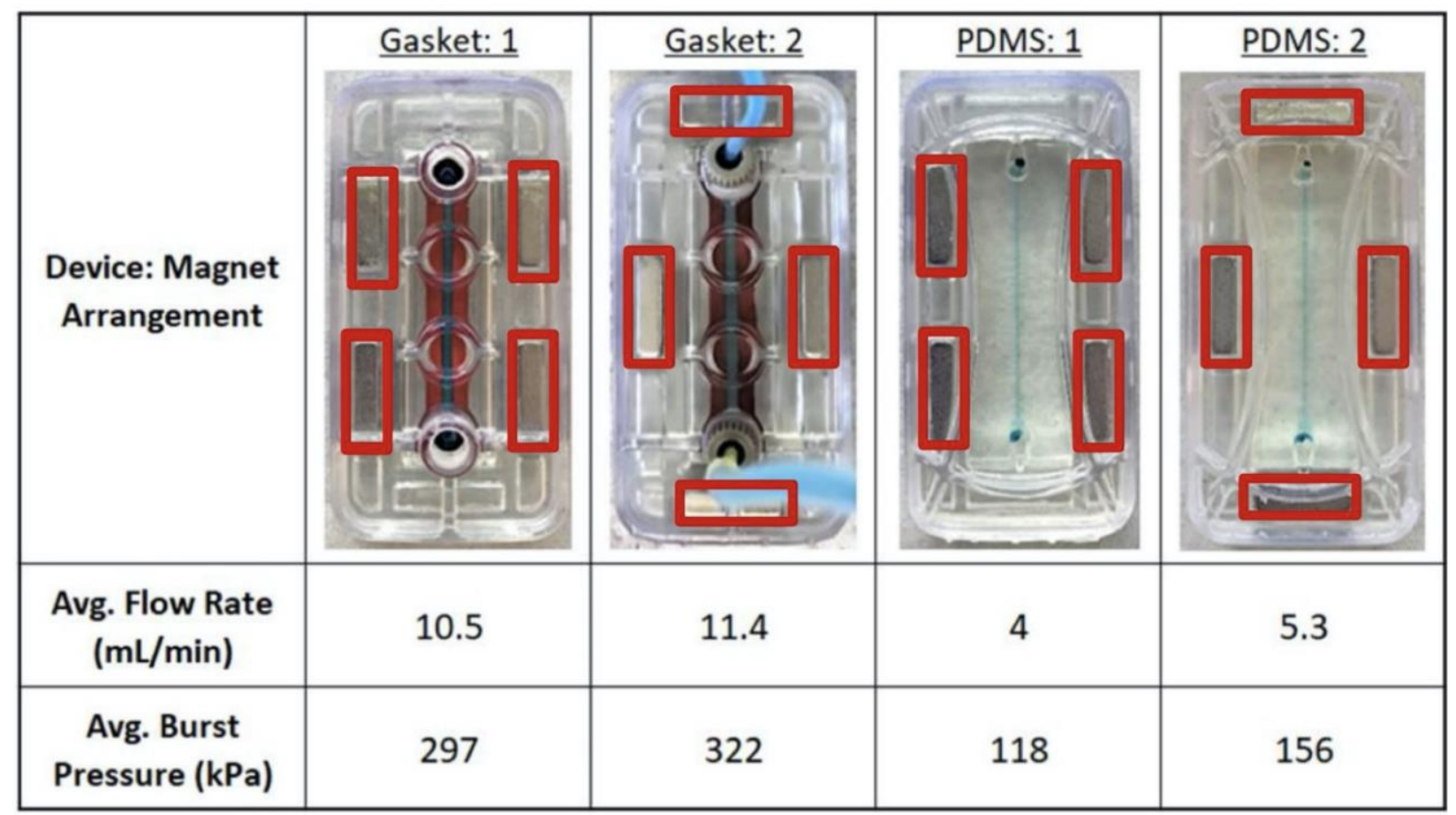

Figure 2. Comparison of the average flow rates and corresponding burst pressures between different magnet arrangements. Burst pressures were not significantly different between "Gasket: 1" and "Gasket: 2". n=4 for all device designs and magnet arrangements.

\section{Electrochemical sensing in the sensor module}

Thermoplastic electrodes (TPEs) in fingertight fittings were successfully integrated into the sensor module device. The ability to add two TPEs to the sensor module allowed for the working and counter electrodes to be adjacent to each other in the device with a reference electrode downstream. This configuration was used to test TPE performance in the sensor module under flow conditions with a ferri/ferrocyanide sample (Figure 3A). The average peak current for $5 \mathrm{mM}$ $\left[\mathrm{Fe}(\mathrm{CN})_{6}\right]^{3-14}$ is statistically similar regardless of flow rate for the rates tested $(\mathrm{p}=0.873$ by oneway ANOVA), supporting that the speed of flow does not significantly impact the peak current observed at the flow rates tested. Data collection was performed with the same two TPEs for the entirety of the experiment with randomized selection of flow rate to reduce possible impacts of electrode surface degradation. However, there is considerable variation in the triplicate sets of measurements at each flow rate (average $\% \mathrm{RSD}=14 \%$ ). It is unlikely that the electrodes were 
degraded because the same electrodes were used on subsequent days for the concentration experiments without the showing the inconsistencies (average \% RSD $=4.6 \%$ ).

Under flow, CVs usually give a sigmoidal shape rather than a peak due to a constant influx of reactants to the electrode surface, ${ }^{40}$ however, peaks are observed in the voltammograms from the sensor module under flow conditions (Figure 3B). The static solution behavior could be due to a small volume of solution becoming trapped at the electrode surface because the electrode is slightly recessed from the flow channel. The small volume then would act more similarly to a static solution for CVs. Future studies will work to alleviate this problem by optimizing the fingertight fitting electrode design.
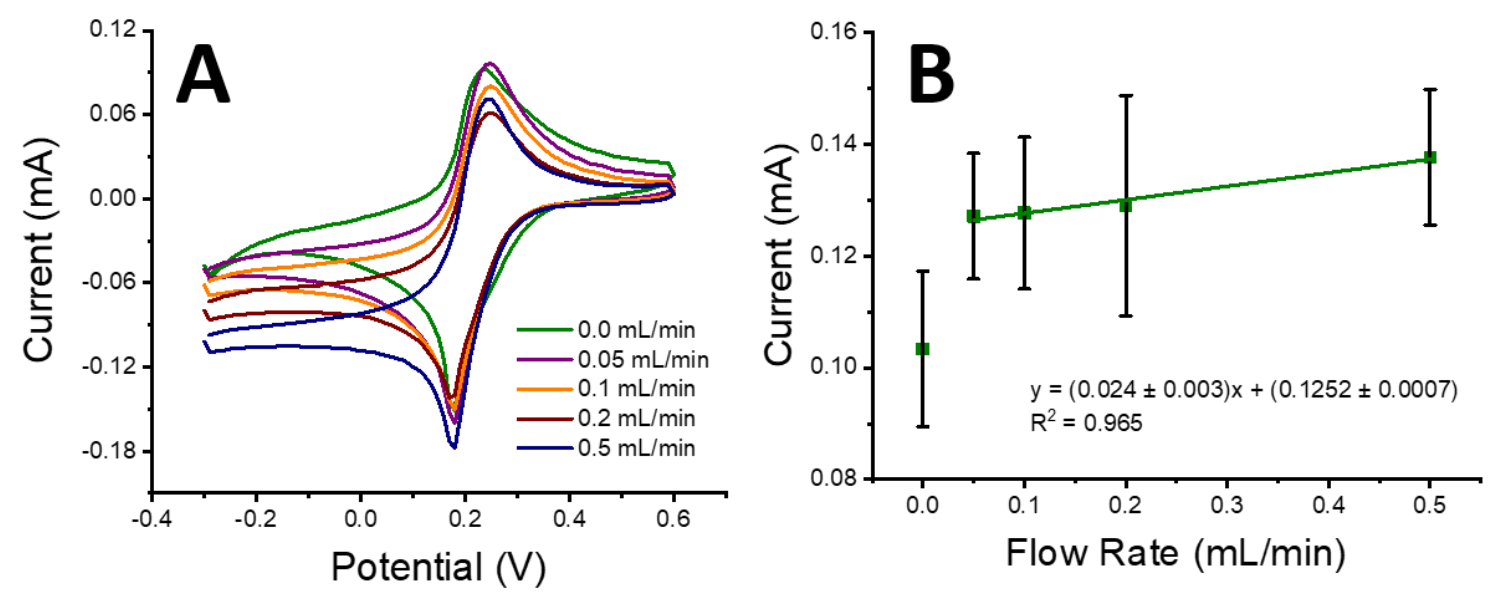

Figure 3. (A) Cyclic voltammetry for $5 \mathrm{mM}\left[\mathrm{Fe}(\mathrm{CN})_{6}\right]^{3-14-}$ in $1 \mathrm{M} \mathrm{KNO}_{3}$ at varying flow rates. (B) Peak currents via cyclic voltammetry from varying flow rate of $5 \mathrm{mM}\left[\mathrm{Fe}(\mathrm{CN})_{6}\right]^{3-14} /\left[\mathrm{Fe}(\mathrm{CN})_{6}\right]^{4-}$ in $1 \mathrm{M} \mathrm{KNO}_{3}$ from 0 $\mathrm{mL} / \mathrm{min}$ to $0.5 \mathrm{~mL} / \mathrm{min}$. Error bars represent the standard deviation of triplicate measurements.

The quantification capability of TPEs in the sensor module was tested via cyclic voltammetry with a redox molecule ferri/ferrocyanide under stopped flow conditions (Figure 4A). The peak current of the CVs produced a linear response with increasing concentration demonstrating that the sensors can be used for quantitative detection (Figure 4B). CVs were taken for proof of concept, but a more sensitive electrochemical technique such as amperometry or square wave voltammetry could also be used.

The ability of the sensor module to accommodate multiple electrochemical sensors simultaneously was shown by integrating two TPE working electrodes with a reference and counter electrode downstream. Ferrocenylmethyl trimethylammonium $\left(\mathrm{FcTMA}^{+}\right.$), a redox molecule with fast kinetics for electrode characterization, was injected and measured amperometrically in flow. FcTMA ${ }^{+}$was oxidized at the upstream working electrode (WE1 in Figure 5A) with a potential of $+400 \mathrm{mV}$ and then reduced at the downstream working electrode (WE2 in Figure 5A) with a potential of $+200 \mathrm{mV}$. The resulting amperograms (Figure 5B) show that the working electrodes function independently and concurrently. The first sample injection resulted in peak oxidation and reduction currents of $0.52 \mu \mathrm{A}$ and $-0.20 \mu \mathrm{A}$, respectively. Subsequent sample injections resulted in consistent peak oxidation and reduction currents of 0.65 $\pm 0.01 \mu \mathrm{A}$ and $-0.240 \pm 0.006 \mu \mathrm{A}$, respectively. The consistency in peak currents after the initial peaks show that the sensor module provides reliable measurements in flow. Successful use of TPEs with $\mathrm{FcTMA}^{+}$and ferri/ferrocyanide opens the door for possibility of applying previously reported 
TPE's with covalent surface modifications, for TPE-based biosensors that could be integrated into the sensor module. ${ }^{36,41}$
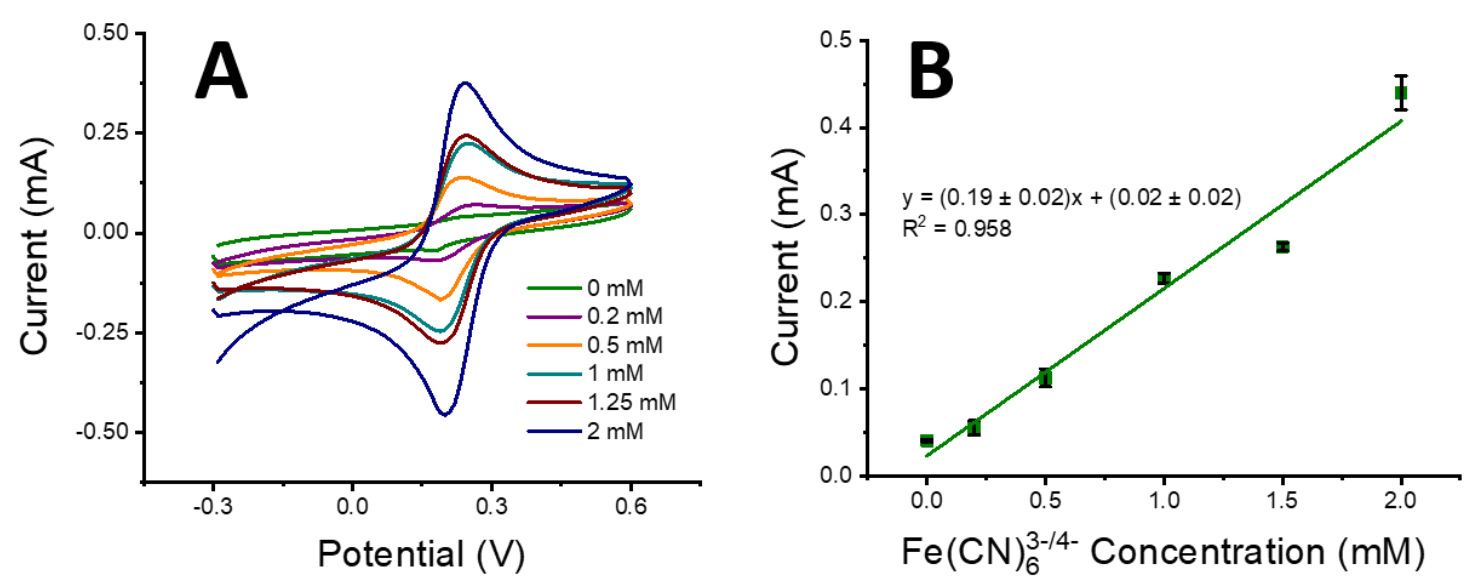

Figure 4. (A) Overlay of cyclic voltammograms for concentrations $0-2.0 \mathrm{mM}$ of $\left[\mathrm{Fe}(\mathrm{CN})_{6}\right]^{3-14-}$ in $1 \mathrm{M} \mathrm{KNO}_{3}$ using TPEs in the sensor module. (B) Linear fit for peak current as a function of concentration of $\left[\mathrm{Fe}(\mathrm{CN})_{6}\right]^{3-/ 4-}$. Error bars represent the standard deviation of $n=3$.
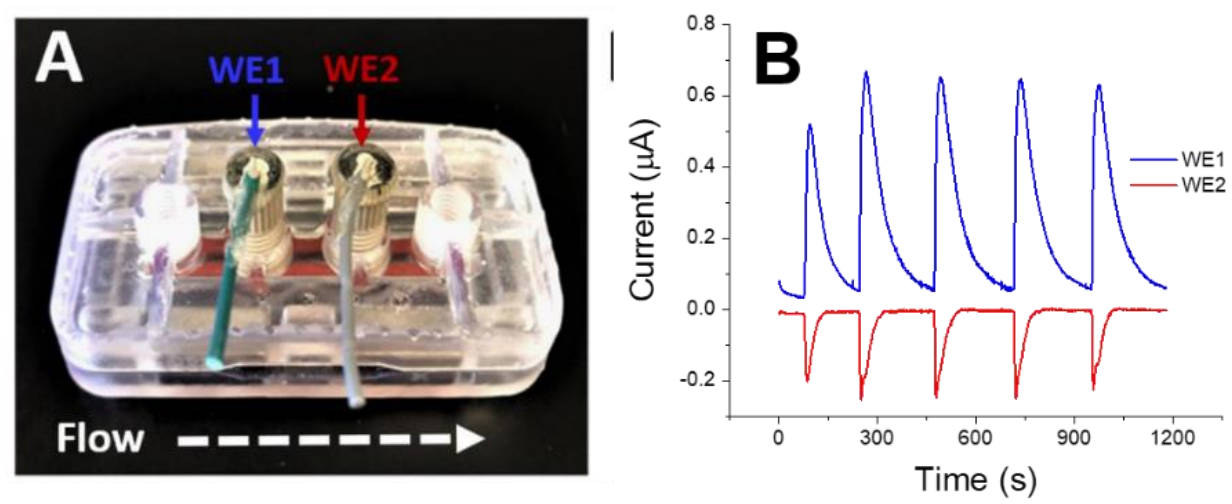

Figure 5. (A) A representative sensor module with two working electrodes in line with the microfluidic channel. (B) Amperogram showing the repeated oxidation (blue peaks) and reduction (red peaks) of FcTMA ${ }^{+}$.

\section{Conclusion}

Herein, the feasibility of a highly user-friendly, magnetically sealed sensor module was established for on-line electrochemical sensing with fluidic devices. Burst pressures were assessed to determine optimal magnet placement prior to testing of fingertight fitting TPEs in the sensor module. Successful detection of ferri/ferrocyanide and $\mathrm{FcTMA}^{+}$in flow through the sensor module provides a proof of concept for integrating electrochemical sensors for multiple biologically relevant analytes such as glucose and lactate. This novel sensing module provides a tool for customizable, plug-and-play format for biosensing when coupled with fluidic systems, such as OOCs. 


\section{References}

1. C. Liu, Y. Qu, Y. Luo and N. Fang, ELECTROPHORESIS, 2011, 32, 3308-3318.

2. A. H. C. Ng, U. Uddayasankar and A. R. Wheeler, Analytical and Bioanalytical Chemistry, 2010, 397, 991-1007.

3. T. Ozer, C. McMahon and C. S. Henry, Annual Review of Analytical Chemistry, 2020, 13, 85109.

4. S. N. Bhatia and D. E. Ingber, Nature Biotechnology, 2014, 32, 760-772.

5. Y. S. Zhang, J. Aleman, S. R. Shin, T. Kilic, D. Kim, S. A. Mousavi Shaegh, S. Massa, R. Riahi, S. Chae, N. Hu, H. Avci, W. Zhang, A. Silvestri, A. Sanati Nezhad, A. Manbohi, F. De Ferrari, A. Polini, G. Calzone, N. Shaikh, P. Alerasool, E. Budina, J. Kang, N. Bhise, J. Ribas, A. Pourmand, A. Skardal, T. Shupe, C. E. Bishop, M. R. Dokmeci, A. Atala and A. Khademhosseini, Proc Natl Acad Sci U S A, 2017, 114, E2293-E2302.

6. X. Li and C. J. Easley, Anal Bioanal Chem, 2018, 410, 791-800.

7. R. K, Biosensors Journal, 2017, 06.

8. P. Shah, J. V. Fritz, E. Glaab, M. S. Desai, K. Greenhalgh, A. Frachet, M. Niegowska, M. Estes, C. Jager, C. Seguin-Devaux, F. Zenhausern and P. Wilmes, Nat Commun, 2016, 7, 11535.

9. M. H. Wu, J. L. Lin, J. Wang and Z. Cui, Biomed Microdevices, 2009, 11, 265-273.

10. S. E. Eklund, D. E. Cliffel, E. Kozlov, A. Prokop, J. Wikswo and F. Baudenbacher, Analytica Chimica Acta, 2003, 496, 93-101.

11. A. Richardson, L. A. Schwerdtfeger, D. Eaton, I. McLean, C. S. Henry and S. A. Tobet, Analytical Methods, 2020, 12, 297-303.

12. Z. Lin, T. Cherng-Wen, P. Roy and D. Trau, Lab Chip, 2009, 9, 257-262.

13. S. Prill, M. S. Jaeger and C. Duschl, Biomicrofluidics, 2014, 8, 034102.

14. J. L. Erkal, A. Selimovic, B. C. Gross, S. Y. Lockwood, E. L. Walton, S. McNamara, R. S. Martin and D. M. Spence, Lab Chip, 2014, 14, 2023-2032.

15. S. A. Gowers, V. F. Curto, C. A. Seneci, C. Wang, S. Anastasova, P. Vadgama, G. Z. Yang and M. G. Boutelle, Anal Chem, 2015, 87, 7763-7770.

16. I. C. Samper, S. A. N. Gowers, M. L. Rogers, D. R. K. Murray, S. L. Jewell, C. Pahl, A. J. Strong and M. G. Boutelle, Lab Chip, 2019, 19, 2038-2048.

17. A. Waldbaur, H. Rapp, K. Länge and B. E. Rapp, Analytical Methods, 2011, 3.

18. L. H. Duong and P. C. Chen, Biomicrofluidics, 2019, 13, 024108.

19. S. Takenaga, B. Schneider, E. Erbay, M. Biselli, T. Schnitzler, M. J. Schöning and T. Wagner, physica status solidi (a), 2015, 212, 1347-1352.

20. M. J. Beauchamp, G. P. Nordin and A. T. Woolley, Anal Bioanal Chem, 2017, 409, 4311-4319.

21. C. S. Carrell, C. P. McCord, R. M. Wydallis and C. S. Henry, Anal Chim Acta, 2020, 1124, 7884.

22. Y. Temiz, R. D. Lovchik, G. V. Kaigala and E. Delamarche, Microelectronic Engineering, 2015, 132, 156-175.

23. C. S. Thompson and A. R. Abate, Lab Chip, 2013, 13, 632-635.

24. X. Gong, X. Yi, K. Xiao, S. Li, R. Kodzius, J. Qin and W. Wen, Lab Chip, 2010, 10, 2622-2627.

25. B. G. Chung, J. W. Park, J. S. Hu, C. Huang, E. S. Monuki and N. L. Jeon, BMC Biotechnol, 2007, 7, 60 .

26. U. Y. Schaff, M. M. Xing, K. K. Lin, N. Pan, N. L. Jeon and S. I. Simon, Lab Chip, 2007, 7, 448456.

27. A. Lamberti, A. Sacco, S. Bianco, E. Giuri, M. Quaglio, A. Chiodoni and E. Tresso, Microelectronic Engineering, 2011, 88, 2308-2310.

28. A. Konda, J. M. Taylor, M. A. Stoller and S. A. Morin, Lab Chip, 2015, 15, 2009-2017.

29. V. V. Abhyankar, M. Wu, C. Y. Koh and A. V. Hatch, PLoS One, 2016, 11, e0156341.

30. E. Tkachenko, E. Gutierrez, M. H. Ginsberg and A. Groisman, Lab Chip, 2009, 9, 1085-1095.

31. M. Rafat, D. R. Raad, A. C. Rowat and D. T. Auguste, Lab Chip, 2009, 9, 3016-3019. 
32. T. Ozer, C. McCord, B. Geiss, D. Dandy and C. S. Henry, Journal of the Electrochemical Society, 2021, 168.

33. S. G. Lemay, D. M. van den Broek, A. J. Storm, D. Krapf, R. M. M. Smeets, H. A. Heering and C. Dekker, Analytical Chemistry, 2005, 77, 1911-1915.

34. D. Qin, Y. Xia and G. M. Whitesides, Nat Protoc, 2010, 5, 491-502.

35. K. J. Klunder, Z. Nilsson, J. B. Sambur and C. S. Henry, Journal of the American Chemical Society, 2017, 139, 12623-12631.

36. K. J. Klunder, K. M. Clark, C. McCord, K. E. Berg, S. D. Minteer and C. S. Henry, Lab on a Chip, 2019, 19, 2589-2597.

37. E. Noviana, K. J. Klunder, R. B. Channon and C. S. Henry, Analytical chemistry, 2019, 91, 24312438.

38. M. Rasponi, F. Piraino, N. Sadr, M. Laganà, A. Redaelli and M. Moretti, Microfluidics and Nanofluidics, 2010, 10, 1097-1107.

39. P. Occhetta, E. Biffi and M. Rasponi, in Microfluidic and Compartmentalized Platforms for Neurobiological Research, ed. E. Biffi, Springer New York, New York, NY, 2015, DOI: 10.1007/978-1-4939-2510-0_2, pp. 25-38.

40. B. J. Adesokan, X. Quan, A. Evgrafov, A. Heiskanen, A. Boisen and M. P. Sørensen, Journal of Electroanalytical Chemistry, 2016, 763, 141-148.

41. K. E. Berg, Y. R. Leroux, P. Hapiot and C. S. Henry, ChemElectroChem, 2019, 6, 4811-4816. 